\title{
Use of integral experiments in support to the validation of JEFF-3.2 nuclear data evaluation
}

\author{
Nicolas Leclaire ${ }^{\mathrm{a}}$, Bertrand Cochet, Alexis Jinaphanh, and Wim Haeck \\ Institut de Radioprotection et de Sûreté Nucléaire, PSN-EXP/SNC, Fontenay-aux-Roses, France
}

\begin{abstract}
For many years now, IRSN has developed its own Monte Carlo continuous energy capability, which allows testing various nuclear data libraries. In that prospect, a validation database of 1136 experiments was built from cases used for the validation of the APOLLO2-MORET 5 multigroup route of the CRISTAL V2.0 package. In this paper, the $\mathrm{k}_{\text {eff }}$ obtained for more than 200 benchmarks using the JEFF-3.1.1 and JEFF3.2 libraries are compared to benchmark $k_{\text {eff }}$ values and main discrepancies are analyzed regarding the neutron spectrum. Special attention is paid on benchmarks for which the results have been highly modified between both JEFF-3 versions.
\end{abstract}

\section{Introduction}

Since the early eighties, the Institut de Radioprotection et de Sûreté Nucléaire (IRSN) has been developing MORET, a Monte-Carlo calculation code for criticality calculations. It was delivered to French criticality safety practitioners within the CRISTAL package [1] in a multigroup version using macroscopic cross sections calculated by the APOLLO2 cell code. A large validation database composed of more than 2500 experiments was established for this package, based on the selection of critical experiments covering most operations of the nuclear fuel cycle. For its $5^{\text {th }}$ release, the MORET code [2] also embeds a continuous energy route, allowing the use of various nuclear data libraries. The continuous energy route has also its own validation database comprising 1136 experiments derived from the 2500 previous ones. It therefore allows contributing to nuclear data validation. In this paper, the JEFF-3.2 library, processed with the IRSN GAIA tool (using NJOY) [3], is tested in the MORET 5 continuous energy validation suite on a selection of more than 200 benchmarks taken from the 1136 available. The results are compared with those obtained with the JEFF3.1.1 evaluation and the differences against benchmark $\mathrm{k}_{\text {eff }}$ are analyzed. Special attention is paid on benchmarks for which the results have been strongly improved or deteriorated.

\section{Calculation code}

The continuous energy version of the MORET 5.C.1 code [2] is used for the calculations. It employs cross sections processed via the GAIA tool for different evaluations, such as JEFF-3.1.1 and JEFF-3.2. The unresolved energy range treatment of cross sections is ensured through a probability table methodology. Other capabilities were implemented in the MORET 5 code, such as sensitivity calculations of $\mathrm{k}_{\mathrm{eff}}$ to nuclear data [4].

a e-mail: nicolas.leclaire@irsn.fr

\section{Methodology}

In order to test the validity of nuclear data, one needs a wide selection of "reference" experiments. For this study, experiments were extracted from the ICSBEP Handbook [5], which is the main source of evaluated criticality experiments in the world. The variety of configurations and labs allows testing a wide range of neutron spectrum, having reliable statistics and highlighting potential experimental biases.

238 experiments were selected in the thermal, epithermal, and fast energy ranges and were assumed, through their diversity of media and configurations, to be representative of the fuel cycle operations. The calculated $\mathrm{k}_{\text {eff }}$ of the benchmark was systematically compared to the benchmark experimental $\mathrm{k}_{\text {eff }}$. When the difference exceeded three times the sum in quadrature of the experimental uncertainties and the Monte Carlo standard deviation, a bias was identified. Otherwise a good agreement was considered between the calculation and the benchmark.

In order to have feedback on nuclear data, the Monte Carlo standard deviation was chosen sufficiently low $(0.00020)$ in comparison with experimental uncertainties. A huge effort was made to select experiments that were not subject to potential biases and which exhibited low experimental uncertainties. As a result, the analysis of C-E (calculation/experiment) discrepancies would help to determine which nuclear data change is responsible for the discrepancy between libraries.

\section{Calculation results}

\subsection{Fast energy range}

Various plutonium (24 cases) and highly-enriched uranium metallic systems (60 cases) were studied in order to evaluate the impact of the JEFF-3.2 evaluation in the fast energy range. For uranium systems, the ${ }^{235} \mathrm{U}$ enrichment varied between 89.5 wt.\% and 97.7 wt.\%. Various

(C) The Authors, published by EDP Sciences. This is an Open Access article distributed under the terms of the Creative Commons Attribution License 4.0 (http://creativecommons.org/licenses/by/4.0/). 


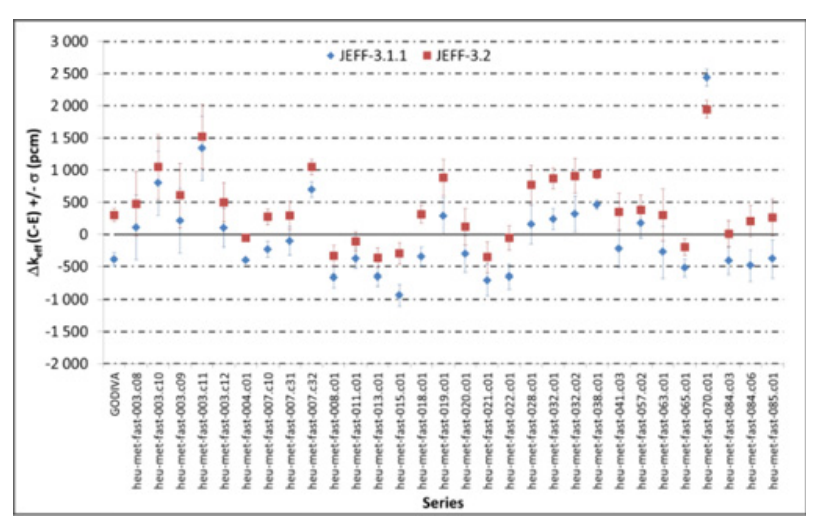

Figure 1. Results for highly enriched uranium (HEU) in fast energy range ( 30 experiments out of 60 studied are reported here).

reflectors were proposed (none, water, depleted uranium, $\mathrm{CH}_{2}$, alumina, tungsten, steel...).

The former under-estimation observed with JEFF-3.1.1 evaluation in Fig. 1 for the unreflected Godiva experiment is no longer observed. This is due to the modifications brought to the ${ }^{235} \mathrm{U}$ nuclear data (prompt and delayed neutron yields, nubar). An increase of $k_{\text {eff }}$ due to ${ }^{235} \mathrm{U}$ nuclear data is also highlighted with water-reflected cases and more generally for all other cases (Fig. 1). However, it does not always lead to an improvement of results, since $k_{\text {eff }}$ results already matched the benchmark $k_{\text {eff }}$ with the JEFF-3.1.1 evaluation. Moreover, some specific tendencies due to the reflectors can be observed, as it is the case for tungsten and lead (HMF-057, -070), for which a compensation effect can be detected. Indeed, the new evaluations of tungsten and lead in JEFF3.2 are responsible for a decrease of $\mathrm{k}_{\mathrm{eff}}$ of around $-800 \mathrm{pcm}$, leading to an improvement of results, which is unfortunately not sufficient to match benchmark $\mathrm{k}_{\text {eff }}$ in the case of HMF-070.c01.

For plutonium systems (Fig. 2), the ${ }^{240} \mathrm{Pu}$ content varied from 1.8 wt. $\%$ to 20.16 wt.\%. Different reflectors were considered (none, water, natural uranium, $\mathrm{CH}_{2}$, alumina, tungsten, steel, etc...). As seen in Fig. 2, compared to JEFF-3.1.1, the new resonance parameters of ${ }^{239} \mathrm{Pu}$ in JEFF-3.2 induce a very slight decrease of $\mathrm{k}_{\text {eff }}$ by less than $100 \mathrm{pcm}$ (see Jezebel experiment). For most configurations, $\mathrm{k}_{\mathrm{eff}}$ is correctly assessed. The ${ }^{240} \mathrm{Pu}$ growing content of plutonium has no significant impact on the trend.

At last, strong effects $(-500 \mathrm{pcm})$ are observed with some reflectors, such as thorium (PMF-008) and tungsten (PMF-005). It is not surprising since the resonance data and nubar of ${ }^{232} \mathrm{Th}$ were modified and a new evaluation for tungsten was proposed in JEFF-3.2. However, for PMF008 , the calculated $\mathrm{k}_{\text {eff }}$ is further from the benchmark $\mathrm{k}_{\text {eff }}$.

It should also be noted that no specific trend is associated to the calculation of the natural/depleted uranium reflector for both plutonium and uranium systems (HMF-028, HMF-032, PMF-006 and PMF-010).

\subsection{Intermediate energy range}

Nine series of benchmarks were retained (HCI-004, HMI001, -006, HMM-002, -003, HCM-001, ICI-001, MCI-005 and BFS experiments).

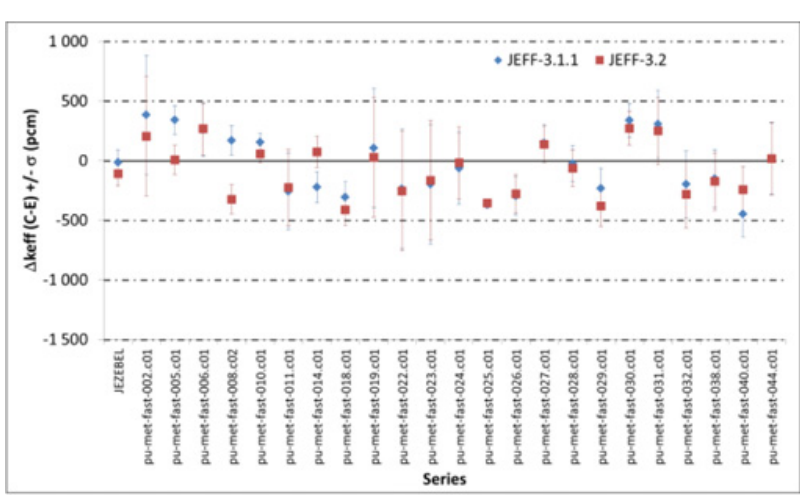

Figure 2. Results for plutonium $(\mathrm{Pu})$ in fast energy range.

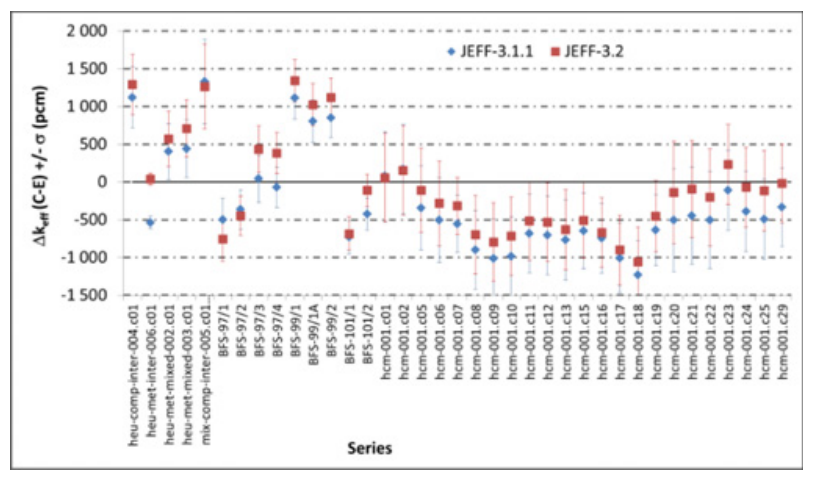

Figure 3. Results for experiments in the intermediate energy range (37 experiments out of 45 reported here).

In order to contribute to ${ }^{235} \mathrm{U}$ nuclear data validation, highly enriched uranium powders were investigated. These critical experiments consisted of cans in variously sized arrays containing enriched uranium dioxide (93\%) with different water content and various interspersed moderation configurations. A $15.24 \mathrm{~cm}$-thickness polyethylene reflector surrounded all configurations. Although categorized as MIXED in ICSBEP Handbook, some experiments also concern the fast and the thermal energy range.

Regarding the validation of $\mathrm{U}, \mathrm{Pu}$ and $\mathrm{Pu}$ in the intermediate energy range, the experimental program performed in BFS reactor by IPPE (Russia) has been investigated [5]. Most of the experiments have been categorized as MIXED, since there are less than 50\% of fissions occurring in fast, intermediate or thermal range.

Figure 3 presents the calculation-experiments discrepancies using both nuclear data evaluations for the nine series (37 experiments) categorized in intermediate energy range.

For highly enriched uranium in epithermal to fast energy range (HCI-004, HMI-006, HMM-002 and -003 and HCM-001), an increase of the calculated $\mathrm{k}_{\text {eff }}$ can be pointed out, improving the results for HCM-001 (see Fig. 3). This is due to the modifications of ${ }^{235} \mathrm{U}$ cross sections in the resolved and unresolved resonance energy ranges and to the nubar. For HMI-001 (not presented in Fig. 3), a larger increase of $\mathrm{k}_{\text {eff }}$ is obtained, which is due to the presence of large amounts of other elements $(\mathrm{Fe}, \mathrm{Cr}$, $\mathrm{Ni}, \mathrm{Al}$ ) in the fissile.

Concerning BFS experiments [6], one can notice that a quite good agreement is obtained, except for experiments with $10 \%$ of ${ }^{240} \mathrm{Pu}(99 / 1$ and 99/2). The 
Table 1. $\mathrm{k}_{\text {eff }}$ results for IEU-COMP-INTER-001.

\begin{tabular}{|l|l|c|c|c|}
\hline Case & $\begin{array}{l}\text { Benchmark } \\
\left(\mathrm{k}_{\text {eff }}(\text { Unc. 1 } \sigma)\right.\end{array}$ & JEFF-3.1.1 & JEFF-3.2 & $\begin{array}{c}\text { Discrepancy } \\
\text { J3.2-J3.1.1 } \\
(\mathbf{p c m})\end{array}$ \\
\hline 1 & $0.969(0.005)$ & 0.99456 & 0.98229 & -1227 \\
\hline 2 & $0.980(0.003)$ & 0.98555 & 0.98236 & -319 \\
\hline 3 & $1.014(0.006)$ & 1.00443 & 1.01926 & 1483 \\
\hline 4 & $0.964(0.012)$ & 0.92127 & 0.93745 & 1618 \\
\hline
\end{tabular}

modification of $k_{\text {eff }}$ entailed by the introduction of the JEFF-3.2 evaluation is correlated to the water content. $\mathrm{k}_{\mathrm{eff}}$ is decreased by $-200 \mathrm{pcm}$ when the water content is equal to zero (97/1) and increased for other cases. This deteriorates the results when compared to the benchmark $\mathrm{k}_{\mathrm{eff}}$. Regarding the significant over predicted results for BFS-99, an experimental bias (on Plutonium composition) cannot be excluded.

ICI-001 series involves a stack of intermediate and highly enriched uranium pellets and thorium pellets arranged at a hexagonal pitch. In cases 3 and 4, polyethylene pellets are added to soften the neutron spectrum.

The results are reported in Table 1, which exhibits a refinement of $\mathrm{k}_{\mathrm{eff}} \mathrm{using}$ the JEFF-3.2 library.

Indeed, for faster energy cases (cases 1 and 2), $\mathrm{k}_{\mathrm{eff}}$ is decreased by $300 \mathrm{pcm}$ to $1200 \mathrm{pcm}$. For intermediate and thermal cases (cases 3 and 4), an increase of $\mathrm{k}_{\text {eff }}$ (around $1500 \mathrm{pcm}$ ) allows improving results, but is not sufficient for case 4 to match the benchmark $k_{\text {eff }}$. These tendencies might be attributed to the nuclear data of ${ }^{232} \mathrm{Th}$.

\subsection{Thermal energy range}

This category gathers experiments involving highly enriched uranium in solution, low-enriched uranium in lattices of rods or mixed $\mathrm{UO}_{2}-\mathrm{PuO}_{2}$ in lattices of rods. As no significant tendency versus evaluation has been identified, for plutonium or lowly enriched uranium, these series are not presented in this paper. 11 experiments with highly enriched uranium solutions $\left({ }^{235} \mathrm{U}\right.$ enrichment higher than $89.3 \mathrm{wt} . \%$ ) with concentrations ranging from $13.24 \mathrm{~g} / \mathrm{l}$ to $516.6 \mathrm{~g} / \mathrm{l}$ and various reflectors (none, light water, heavy water, beryllium oxide) were investigated to test the modification of the ${ }^{235} \mathrm{U}$ cross sections in the resonance range. Figure 4 shows the calculationexperiment discrepancies for both evaluations.

For solutions in light water, no significant impact of the evaluation is observed. In particular, badly estimated $\mathrm{k}_{\mathrm{eff}}$ results with JEFF-3.1.1 are not improved with JEFF3.2 and configurations without absorbers still exhibit $\mathrm{k}_{\mathrm{eff}}$ results consistent with the benchmark (HST-001.c01 and HST-049-c01). For solutions in heavy water (HST-004 and HST-020), which implies a hardener neutron spectrum, an increase of $k_{\text {eff }}$ of around 500-1000 pcm is observed with the JEFF-3.2 evaluation.

Figure 5 shows Calculation-experiments discrepancies obtained with both evaluations for $\mathrm{UO}_{2}$ lattices.

For uranium involved in lattices of $\mathrm{UO}_{2}$ rods in water (LCT-007), quite marginal discrepancy between libraries is observed. It was expected since the data are taken from the JEFF-3.1.1 evaluation with insignificant modifications. The main discrepancy observed comes from the structural

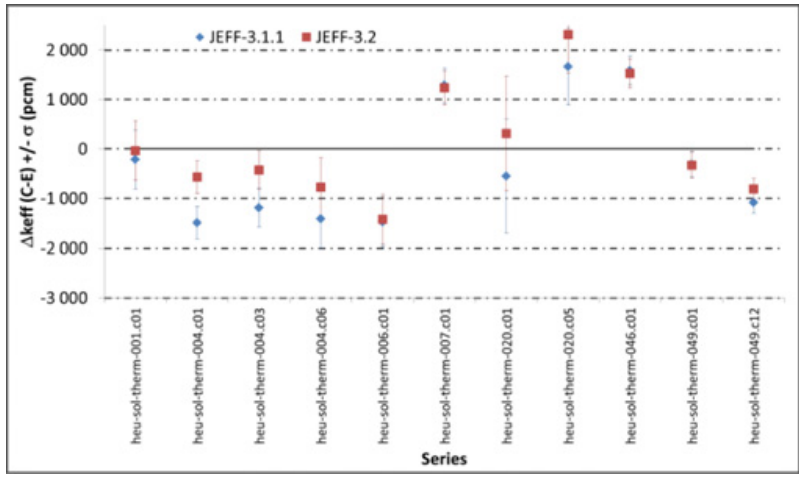

Figure 4. Results for highly enriched uranium (HEU) in solution.

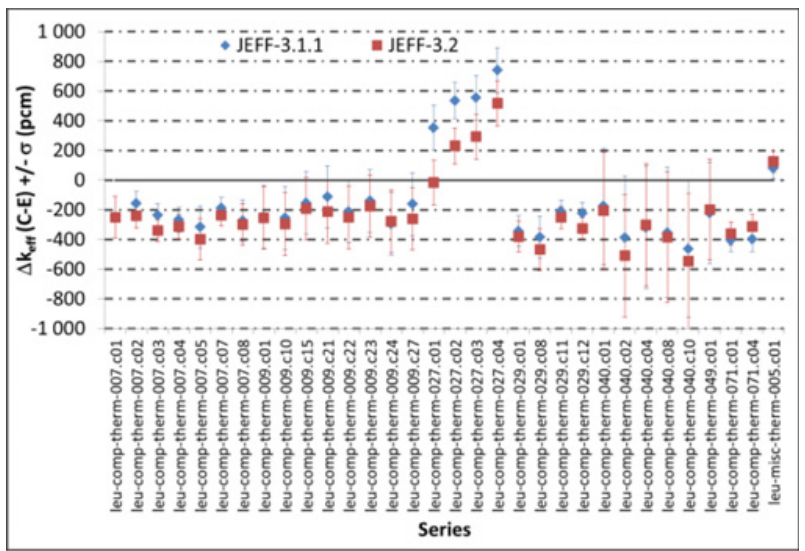

Figure 5. Results for lowly enriched uranium (LEU) in lattices of rods (32 experiments out of 69 reported here).

materials involved in the absorbing canisters or in the reflectors surrounding the lattices.

In particular, for LCT-027 (lead reflector), one can see a decrease of $k_{\text {eff }}$ with the JEFF-3.2 evaluation, improving the $\mathrm{k}_{\mathrm{eff}}$ results. It is mainly due to the cross sections of lead in thermal and epithermal energy range. Indeed, for JEFF3.2 evaluation, the resonance parameters were modified for all isotopes of lead and inelastic cross sections replaced with other evaluations for ${ }^{207} \mathrm{~Pb}$ and ${ }^{208} \mathrm{~Pb}$. However, for large thicknesses of reflector, $\mathrm{k}_{\mathrm{eff}}$ is still overestimated, which suggest that the modifications are not sufficient.

Concerning lattices of mixed $\mathrm{UO}_{2}-\mathrm{PuO}_{2}$ rods in water (32 experiments), the selected benchmarks exhibit $\mathrm{Pu} / \mathrm{UPu}$ ratios varying between $3 \mathrm{wt} . \%$ and $22 \mathrm{wt} . \%,{ }^{240} \mathrm{Pu}$ contents varying between $8.5 \mathrm{wt} . \%$ and $22.2 \mathrm{wt} \%$. The moderator to fuel ratio is comprised between 1.7 and 17.5. Results are reported on Fig. 6.

For high plutonium contents (MCT-001 and -003), one can notice that the JEFF-3.2 evaluation increases $k_{\text {eff }}$, contributing to an improvement of results (see Fig. 6). A tendency versus moderation to fuel ratio can also be stressed, better agreement being obtained for low and high moderation to fuel ratios.

\subsection{Other issues}

In this section, attention is focused on experiments showing great impact of JEFF-3.2 nuclear data.

The first series deals with BIG TEN experiment involving intermediate enriched uranium $(10 \mathrm{wt} . \%)$ in fast energy spectrum reflected by a thick ${ }^{238} \mathrm{U}$ reflector. 


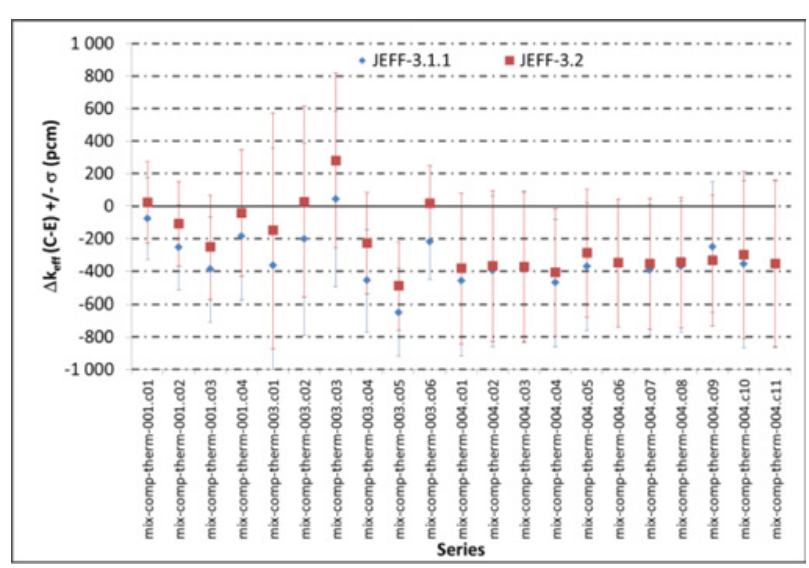

Figure 6. Results for lattices of mixed $\mathrm{UO}_{2}-\mathrm{PuO}_{2}$ rods in water.

Table 2. $\mathrm{k}_{\text {eff }}$ results with JEFF-3.1.1 and JEFF-3.2 evaluations $\left(\sigma_{\mathrm{MC}}=0.00020\right)$.

\begin{tabular}{|c|c|c|c|c|c|}
\hline \multirow{3}{*}{ Case } & \multirow{3}{*}{$\begin{array}{c}\text { Benchmark } \\
\mathbf{k}_{\text {eff }} \\
(\text { Unc. 1 } \sigma)\end{array}$} & \multicolumn{4}{|c|}{$\mathbf{k}_{\text {eff }}$} \\
\hline & & \multirow{2}{*}{\begin{tabular}{|c|} 
JEFF- \\
3.1.1 \\
All \\
isotopes
\end{tabular}} & \multicolumn{3}{|c|}{ JEFF-3.2 } \\
\hline & & & $\begin{array}{c}\text { All } \\
\text { isotopes }\end{array}$ & \multicolumn{2}{|c|}{$\begin{array}{c}\text { Only isotope of } \\
\text { interest }\end{array}$} \\
\hline \multirow{3}{*}{$\begin{array}{c}\text { IMF- } \\
\text { 007.c01 } \\
\text { (BIG } \\
\text { TEN) }\end{array}$} & \multirow{3}{*}{$\begin{array}{c}1.00480 \\
(0.00020)\end{array}$} & \multirow[b]{3}{*}{0.99783} & \multirow{3}{*}{1.00359} & \multirow{3}{*}{\begin{tabular}{|c|}
${ }^{238} \mathrm{U}$ \\
${ }^{235} \mathrm{U}$ \\
${ }^{238} \mathrm{U}+$ \\
${ }^{235} \mathrm{U}$
\end{tabular}} & 1.00429 \\
\hline & & & & & \multirow[b]{2}{*}{1.00536} \\
\hline & & & & & \\
\hline $\begin{array}{c}\text { HCT- } \\
021 . c 01\end{array}$ & $\begin{array}{c}1.00080 \\
(0.00290)\end{array}$ & 0.99280 & 0.99680 & ${ }^{232} \mathrm{Th}$ & 0.99558 \\
\hline $\begin{array}{l}\text { U233- } \\
\text { MF-001 }\end{array}$ & $\begin{array}{c}1.00000 \\
(0.00100)\end{array}$ & 1.00639 & 0.99996 & ${ }^{233} \mathrm{U}$ & 0.99986 \\
\hline
\end{tabular}

The second series of interest is the HEU-COMPTHERM-021, where $\mathrm{UO}_{2}-\mathrm{ThO}_{2}$ rods are arranged in lattices in light water. Uranium is enriched at $93.15 \mathrm{wt} . \%$ in ${ }^{235} \mathrm{U}$. The atom ratio of thorium over uranium is equal to 15 .

The third one addresses ${ }^{233} \mathrm{U}$ issues in fast energy spectrum. Uranium is more than $90 \mathrm{wt} . \%$ enriched in ${ }^{233} \mathrm{U}$. It takes the shape of a metallic sphere.

The $\mathrm{k}_{\mathrm{eff}}$ results versus evaluation are reported in Table 2. JEFF-3.1.1 is the major evaluation for all elements except for the material of interest, where JEFF-3.2 is also used.

One can see that, for the 3 benchmarks, JEFF-3.2 leads to better results than JEFF-3.1.1. For BIG TEN, the two isotopes ${ }^{235} \mathrm{U}$ and ${ }^{238} \mathrm{U}$ contribute to an increase of $\mathrm{k}_{\mathrm{eff}}$. Combining the effect of the two isotopes allows matching the benchmark $\mathrm{k}_{\mathrm{eff}}$.

For ${ }^{232} \mathrm{Th}$, in the thermal energy range, one can notice the improvement brought by the JEFF-3.2 evaluation in the $\mathrm{k}_{\mathrm{eff}}$ result, with a $\mathrm{k}_{\text {eff }}$ increase. This was already observed for the ICI-001 series in the same energy range.

For ${ }^{233} \mathrm{U}$, in the fast energy range, the new evaluation of ${ }^{233} \mathrm{U}$ leads to a $-700 \mathrm{pcm}$ decrease of $\mathrm{k}_{\mathrm{eff}}$, allowing improvement of $\mathrm{k}_{\mathrm{eff}}$ result.

\section{Conclusion}

Based on a selection of 238 experiments spread on the whole energy spectrum and covering most operations of the fuel cycle, IRSN tested the new JEFF-3.2 evaluation with the MORET 5.C.1 Monte Carlo continuous energy code.

In thermal energy spectrum, as JEFF-3.1.1 already gives satisfactory results for fissile media, the main improvement is associated to the treatment of structural materials, such as lead in transportation casks or heavy water. Solutions with plutonium, lowly and highly enriched uranium are not subject to significant changes. The same conclusion can be drawn for lattices of $\mathrm{UO}_{2}$ rods in water without absorbing canisters or specific reflector. Moreover, the under-estimation of $\mathrm{k}_{\text {eff }}$ obtained with JEFF3.1.1 for thorium cross sections is partly corrected with the JEFF-3.2 evaluation.

In the fast energy range, the results for plutonium are not significantly affected by the modification of nuclear data. However, it is not the case for highly enriched uranium, for which a significant increase of $\mathrm{k}_{\mathrm{eff}}$ is obtained, correcting the $500 \mathrm{pcm}$ under-estimation pointed out with JEFF-3.1.1 for highly enriched uranium reflected or not by water.

It should also be noted that a decrease of $\mathrm{k}_{\mathrm{eff}}$ is obtained for tungsten and thorium reflectors, resulting in an improvement of the results. Nevertheless, some improvements are still needed in the case of tungsten.

Additionally, the new ${ }^{233} \mathrm{U}$ cross sections allow better agreement with the benchmark $\mathrm{k}_{\mathrm{eff}}$ in fast energy spectrum, reducing by $0.6 \%$ the previous over-estimation of $\mathrm{k}_{\text {eff }}$.

Finally, it should be reminded that this analysis is based on a restricted number of experiments. Further analyses will have to be based on an extended set of experiments to better address the requirements of the validation process and will also use sensitivity studies.

The authors would like to acknowledge Isabelle Duhamel for its support in the analysis of the results.

\section{References}

[1] E. Gagnier, F.X. Giffard, C. Riffard, C. Carmouze, N. Leclaire, I. Duhamel, "Experimental validation of the CRISTAL V2.0 French criticality calculation package", ICNC2015, Charlotte, p. 813-823, 2015

[2] B. Cochet, A. Jinaphanh, L. Heulers, O. Jacquet "Capabilities overview of the MORET 5 Monte Carlo code", Annals of Nuclear Energy 82, 74-84 (2015)

[3] W. Haeck, "GAIA User's Manual Version 1.0.0", PSN-EXP/SNC/2015-165

[4] A. Jinaphanh, N. Leclaire, B. Cochet, "ContinuousEnergy Sensitivity Coefficients in the MORET Code", Nuclear Science and Engineering, Volume 184, Number 1, September 2016

[5] International Handbook of Evaluated Criticality Safety Benchmark Experiments, Organization of Economic Cooperation and Development-Nuclear Energy NEA/NSC/DOC(95)03, September 2014 Edition

[6] T. Ivanova et al., "Impact of Nuclear Data on Multiplication Factor and Reactor Physics Parameters Calculation for Experiments Simulating Damp MOX Powders", ND2007, Nice, 2007 